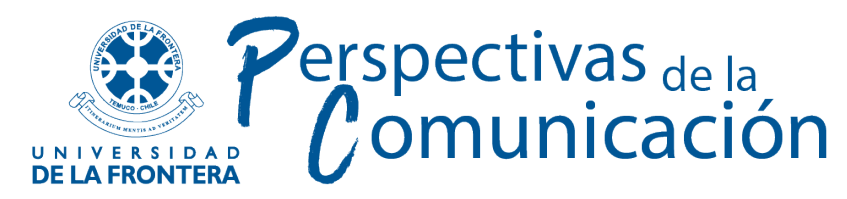

ISSN 0718-4867

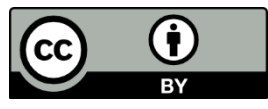

Artículo

\title{
EL PERIODISTA COMO COMUNICADOR ESTRATÉGICO: ¿UN NUEVO MITO DEL PERIODISMO? ${ }^{1}$
}

https://doi.org/0O.0OO/COO-202O-O1

Lic. Camilo Solís

Univ ersidad de Chile, Santiago, Chile

camilo.solis@ug.uchile.cl

ORCID iD: https://orcid.org/0000-0001-5572-3909

Recibido el 28 de febrero de 2019

Ac eptado el 5 de septiembre de 2019

\section{Resumen}

Esta investigación tiene por objetivo observar si los discursos que plantean al periodista como un comunicador estratégico, en el contexto contemporáneo, involucran una superación de aquellos discursos que han sustentado y explicado la labor de los periodistas en nuestra sociedad de manera tradicional. Nos referimos a aquel relato basado en valores como la objetividad, la independencia, el rol fiscalizador del poder -entre otros-, que bien podríamos considerar un mito siguiendo a los autores estudiados en la construcción teórica de esta investigación. ¿Supone la comunicación estratégica una superación de este mito liberal del periodismo? Para esto se analizaron los discursos de académicos que imparten asignaturas sobre comunicación estratégica en escuelas de periodismo de Santiago de Chile. Los principales resultados señalan que efectivamente se observa un desplazamiento parcial de este mito liberal del periodismo para dar paso a discursos que se encuentran en mayor sintonía con un contexto contemporáneo. Este "nuevo mito", vinculado a la comunicación

1 Este artículo informa sobre la investigación denominada Nuevas elaboraciones míticas, el periodista como comunicador est ratégico, la cual fue realizada en el seminario de investigación "Transformaciones culturales del periodista”. Este seminario fu e guiadopor el prof. Hans Stange Marcus en el Institutode la Comunicación e Im agen de la Universidad de Chile. 
estratégica, les entrega a los periodistas una nueva forma de explicar su rol en la sociedad, a la vez que les permite migrar a mejores y más puestos laborales, exigiendo de estos profesionales una adecuación a nuevas formas de ejercicio de la profesión.

Palabras clave: mito, periodismo, comunicación estratégica, comunicador estratégico. 


\title{
THE JOURNALIST AS A STRATEGIC COMMUNICATOR: A NEW JOURNALISM MYTH?
}

\begin{abstract}
The purpose of this research is to observe whether the discourses which present the journalist as a strategic communicator, in the contemporary context, involve overcoming other discourses which have supported and explained the work of journalists in our society in a traditional way. We refer to the narrative based on values such as objectivity, independence, the supervisory role of power-among others-, which could well be considered a myth following the authors studied in the theoretical construction of this research. Is strategic communication an overcoming of this liberal myth of journalism? For this purpose, the discourses of academics who teach subjects on strategic communication in journalism schools of Santiago de Chile were analyzed. The main results indicate that there is indeed a partial displacement of this liberal myth of journalism to make way for discourses that are more in tune with a contemporary context. This new myth, linked to strategic communication, gives journalists a new way of explaining their rolein society, while allowing them to migrate to more and better jobs, requiring of these professionals an adaptation to new forms of exercise of the profession.
\end{abstract}

Keywords: myth, journalism, strategic communication, strategic communicator. 


\section{Introducción}

Es posible observar que las labores que realizan los periodistas como comunicadores corporativos/organizacionales/estratégicos, en el mercado laboral contemporáneo, no siempre calzan con el catálogo de valores tradicionales del periodismo - basado en la objetividad, la independencia, la neutralidad, la fiscalización, entre otros valores. Es posible que estos comunicadores estratégicos realicen su ejercicio profesional en una gama de aplicaciones prácticas mucho más amplia que el periodismo tradicional, realizando su labor sin observar necesariamentelosvalores antes mencionados. Desde esta reflexión inicial, nos preguntamos si los discursos que sostienen a estas nuevas aplicaciones prácticas del periodismo - sobre comunicación estratégica y organizacional-constituyen un relato capaz de explicar la labor de los periodistas en el contexto contemporáneo, de tal forma que signifique una eventual superación de los discursos tradicionales sobre periodismo.

Para el desarrollo de esta idea, tomaremos lo trabajado por algunos autores (Rodríguez, 1998; San Martín, 2008; Salinas y Stange, 2014), quienes plantean la idea de que los valores tradicionales asociados a la cultura profesional de los periodistas -o algunos aspectos de ella-constituyen más bien un mito que, de manera flagrante, dista del ejercicio práctico de la profesión en la actualidad. De la misma forma, a partir de lo planteado por autores como Habermas (1981), Monzón (1985, 2006) y Siebert (1984) observamos que los elementos valóricos fundamentales de este mito -independencia, objetividad, rol fiscalizadorconstituyen un mito liberal del periodismo, pues provienen de prerrogativas liberales y modernas propias de la Ilustración, ocurrida en occidente entre los siglos XVIII y XIX. De esta forma, a partir de la observación de los principales elementos constitutivos de un mito (Barthes, 1999; Lévi-Strauss, 1995), observaremos si los discursos que plantean al periodista como comunicador estratégico u organizacional en el contexto contemporáneo suponen un mito que desplaza a este denominado mito liberal del periodismo.

Desde este problema, nos planteamos la siguiente hipótesis de trabajo: El mito liberal del periodismo carece de rendimiento para explicar completamente el rol de los periodistas en contexto contemporáneo, de tal forma que los discursos que plantean al periodista como comunicador estratégico operan como un nuevo relato mítico que explica el rol del periodista en el contexto actual, tensionando los conceptos del mito liberal del periodismo. 
Como observaremos en el desarrollo teórico de este artículo, hay elementos suficientes como para señalar que estos valores ya han sido - al menos parcialmente- dejados de lado en la práctica, debido principalmente a cambios sobre el contexto en que operan los periodistas. Sin embargo, en lo discursivo, este mito liberal del periodismo sigue presentando vigencia para justificar el rol de los periodistas en la sociedad.

Constatar la existencia de nuevos mitos con capacidad explicativa suficiente para comprender el rol del periodista en tiempos contemporáneos implica cierta superación discursiva de este catálogo de valores del periodismo tradicional. Eventualmente, el surgimiento de un mito que busca explicar el rol de los periodistas en nuevas aplicaciones prácticas - como lo es la comunicación organizacional- podría relacionarse con una necesidad de justificar discursivamenteel hecho de que los periodistas estén migrando hacia nuevas labores y puestos de trabajo. Esto constituye un elemento de gran relevancia pues implicaría repensar el periodismo en términos formativos; principalmente, sobre cómo se enseña y sobre cómo se va a enseñar en tiempos venideros.

\section{Marco Teórico}

\section{El mito moderno}

Hablaremos de mito moderno para alejarnos de la noción clásica de mito relacionada con el relato maravilloso más bien cercano a la teología. En una primera aproximación a esta noción, Barthes (1999: 108) postula que el mito se puede entender como un habla en tanto mensaje, en tanto constituye un "decir". El mito es una realidad dicha y como tal no es un objeto. Se trata más bien de una forma de decir un objeto, es decir: un modo del objeto. Ahora bien, según el autor mencionado, lo propio de este "decir" es que no sea un "decir" aislado, sino que se encuentra investido de su contexto: una historia particular, una sociedad:

(...) el mito constituye un sistema de com unicación, un mensaje. Esto indica que el mito no podría ser un objeto, un concepto o una idea; se trata de un modo de significación, de una forma. Más adelante habrá que imponer a esta for ma límites históricos, condiciones de empleo, reinvestir en ella la sociedad; (...) si el mito es un habla, todolo que justifique un discurso puedeser mito. (...) Cada objeto del mundo pu ede pasar de una existencia cerrada, muda, a un estado or al, abierto a la apropiación de la sociedad, pues ninguna ley, natural o no, im pideh ablarde las cosas. (Barthes,1999: 108)

Pers pectivas de la Comunicación - Vol. 13 - No2 - 2020 - pp. 73-103

Universidad de la Frontera - Chile 
Es el contexto en que se produce este "decir" el que nos permite observar al mito como una existencia no naturaly, más bien, determinada por las condiciones particulares de la historia en que se habla un objeto determinado. A decir de Barthes: "la mitología sólo puede tener fundamento histórico, pues el mito es un habla elegida por la historia: no surge de la 'naturaleza' de las cosas" (Barthes, 1999:108).

Para demostrar esto, el autor realiza un análisis semiológico basado en la estructura formal de cualquier propuesta de sentido, la cual consta de: un significante, un significado y un signo. En esta estructura, el significante es el elem ento vacío sobre el que recae el significado; el significado constituye el sentido que se le otorga a ese significante; $y$, finalmente, el signo "constituye el total asociativo de los dos primeros términos" (1999: 110). Barthes utiliza este análisis para señalar que el mito es fundamentalmente un "sistema semiológico segundo". Es decir, el mito es un sistema semiológico que ocupa como significante - primer elemento del sistema - al signo proveniente de un sistema semiológico previo:

\footnotetext{
El mito es un sistema particular por cuanto se edifica a partir de una cadena semiológica que existe prev iamente: es un sistema semiológico segundo. Lo que con stituye el signo(...) en el prim er sistema, se v u elve simple significante en el segundo. Recordemos a quí que las m aterias del habla mítica (lengua propiamente dicha, fotografia, pintura, cartel, rito, objeto, etc.), por diferentes que sean en un principio y desde el momento en que son captadas por el mito, se reducen a una pura función significante (Barthes, 1999: 111).
}

En este punto, Barthes señala que el resultado del primer sistema semiológico: el signo; ya constituye un sistema completo, ya propone un sentido acabado, el cual "postula un saber, un pasado, una memoria, un orden comparativo de hechos, de ideas, de decisiones" (1999: 113). Sin embargo, el mito toma esta elaboración dotada de sentido y la convierte en un significante vacío, en una forma dispuesta a ser significada por un segundo sistema de significación. Esto supone que el sentido deslizado en el significante del mito constituiría una naturalización de aquel sentido. Se da por hecho ese sentido deslizado. El mito es la naturalización de un sentido:

En el sistem a segundo (mítico), la causalidades artificial, falsa, per o se desliza de alguna manera en los furgones de la naturaleza. Por eso el mito es vivido como una palabra inocente; no porque sus intenciones sean ocultas (si fueran ocultas, no podrían ser eficaces), sino porque están naturalizadas (1999: 121). 
En este sentido, Barthes señala que lo propio del mito es el robo de lenguaje: “¿Qué es lo específico del mito? Es transformar un sentido en forma. Dicho de otro modo, el mito es siempre un robo de lenguaje" (1999: 122). A raíz de lo anterior, Barthes plantea que el mito es un habla despolitizada (1999: 129), puesto que en la medida que naturaliza una propuesta de sentido deslizándola en el significante, da por hecha la operación semiológica anterior, desligándola así de su contexto, de su historia, de su fundamento como lenguaje. El autor plantea que para llegar a esta conclusión es necesario incurrir en una conceptualización amplia de lo que significa lo político: “(...) es necesario entender política en el sentido profundo, como conjunto de relaciones humanas en su poder de construcción del mundo" (Barthes, 1999: 129). En suma, lo propio del mito es la naturalización de un sentido y, por lo tanto, la despolitización del habla debido a una desconexión del lenguaje con su contexto:

El mitononiega las cosas, su función, por el contrario, es hablar de ellas; simplemente las purifica, las v uelve inocentes, las funda com o naturaleza y eternidad, les confiere una claridad que no es la de la explicación, sino de la com probación: si com pruebola im perialidad francesa sin explicarla, estoy a un paso de encontrarla natural, que cae por su peso; me quedotranquilo (Barthes, 1999: 129).

Desde otra vertiente, Lévi-Strauss (1995), observa la presencia recurrente de categorías binarias opuestas en los mitos, es decir, antinomias: "el pensamiento mítico procede de la toma de conciencia de ciertas oposiciones y tiende a su mediación progresiva" (Lévi-Strauss, 1995: 246). El autor señala que los extremos de estas oposiciones consisten en una "estructura simétrica pero invertida", de tal forma que se introduce "un principio de orden allí donde solo existía el caos (...)" (1995: 246). A propósito de lo planteado por Lévi-Strauss, Fernández (1996), señala que esta forma de observar los mitos tiene que ver con "un mecanismo que cuando se enfrenta con la producción de una determinada idea procede mediante el planteamiento y 'resolución' o 'mediación' de conjuntos enteros de oposiciones lógicas entre dos elementos" (Fernández, 1996: 12).

Por su parte, Schoenbach (2001) señala: "Levi-Strauss tells us that typical for the clear-cut structure of myths is their 'binarism' - thejuxtaposition of two principles, such as clever and stupid, big and small, high and low, arrogant and modest, and, of course, good and bad, the 
basic Manicheism that many myths share"2 (Schoenbach, 2001: 363). El mismo autor define "mito" como: "simple, but plausible and poetic, explanations of the world"3 (2001: 361). Como vemos, este elemento antinómico propio de los mitos entrega a los sujetos de una comunidad elementos cognitivos que le permiten situarse y orientarse en su contexto. Esa es la razón por la cual los autores observados conciben al mito como una explicación del mundo, o como una estructura que sienta orden allí donde antes no lo existía. A propósito de esto, Fernández (1996), en la lectura que hace de Burridge (vid. 1968) señala: "Burridge ve la Cultura y el Mito como 'dos formas colectivas' que se oponen entre sí como tesis, antítesis para producir una síntesis que sería, presumiblemente, la comprensión de su cultura por parte de los integrantes de la misma” (Fernández, 1996: 15-16).

Según lo visto, Barthes entrega tres elementos principales para definir al mito moderno. El primer elemento constitutivo del mito es que no se trata de un objeto, sino que pertenece al dominio del habla: un mito siempre es algo que se dice de un objeto y no el objeto mismo. El segundo aporte de Barthes a esta conceptualización dice relación con el componente político que posee el mito: se trata de un habla despolitizada, en la medida que naturaliza un sentido y lo desliza desprovisto de su historia y contexto utilizando un significante ya dotado de sentido. En tercer lugar, Barthes señala que la vehiculización de un mismo concepto a partir de distintas formas pone en evidencia al mito: "la insistencia de una conducta es la que muestra su intención" (1999: 115). De esta forma, instala el criterio de la reiteración insistente de una discursividad, para la verificación de la presencia de un habla mítica. Por otra parte, Lévi-Strauss aporta al concepto del mito con componentes de tipo cognitivo. Según este autor, el mito contiene de manera estructural lógicas binarias o antinómicas que le permiten a un miembro de la comunidad entender y entenderse en su contexto. Este elemento es reforzado por las lecturas que Schoenbach (2001) y Fernández (1996) realizan sobre el mito.

2 Levi-Strauss nos dice que lo típico para la estructura definida de los mitos es su 'binarismo' -la yuxtaposición de dos principios, como inteligente y estúpido, grande y chico, alto y bajo, arrogante y modesto, y, por supuesto, bueno y malo, el Ma niqueísmo básico que muchos mitos comparten. T. del A.

3 Simples, peroplausiblesy poéticas, explicaciones del mundo. T. del A 


\section{El 'mito liberal del periodismo' y su extraña vigencia}

Algunos autores señalan que los valores -o algunos de ellos- másimportantes de la cultura profesional de los periodistas constituyen un mito (Salinas y Stange, 2009, 2014; San Martín, 2008; Rodríguez, 1998). Esta afirmación procede principalmente desde la constatación de que dichos valores tradicionales - objetividad, independencia, fiscalización al poder, entre otros - no se condicen necesariamente con la forma en que los periodistas ejercen su profesión en la cotidianeidad. Es decir, pertenecen al plano discursivo más que práctico. Para esto, es interesante identificar desde dónde proviene este catálogo de valores y prerrogativas tradicionales.

\section{Teoría liberal del periodismo}

La mayoría de las ideas y conceptos que se tienen respecto de la actividad profesional del periodismo, tienen un origen remoto que encuentra sus fundamentos en la instalación del discurso ilustrado y liberal que entre los siglos XVIII y XIX da comienzo al surgimiento de Estados Nacionales en occidente. Este es el contexto en el que surgela prensa como artefacto revolucionario capaz de reproducir y transmitir las ideas del progreso, la libertad y la razón. Estas afirmaciones pueden sostenerse en las lecturas de autores como Habermas (1981), Monzón (1985, 2006) y Siebert (1984), quienes desarrollan la idea de la formación de la opinión o esfera pública, como un fenómeno propio de las ideas modernas de la burguesía, pues "no hay que olvidar que la burguesía frente a la aristocracia se encontraba en un status inferior, de aquíla preocupación delos liberales por cambiar las leyes para llegar a la libertad e igualdad «de derecho»" (Monzón, 1985: 95). A propósito de esto, Monzón señala queluego de la Revolución Francesa "el público políticamente raciocinante es indirectamente reconocido en su función de crítica pública por un discurso de Fox ante la Cámara de los Comunes. Por primera vez se habla en el Parlamento de public opinion en el estricto sentido de esta locución” (Monzón, 1985: 85-86).

El surgimiento de esta opinión pública debe gran parte de su fundamento discursivo al atributo humano de la razón, el cual es considerado el vehículo por el cual se puede alcanzar la verdad. En miras de asegurar la posibilidad de que el ser humano consiga acceder a la verdad es que se consagra, entre otros, el derecho a la libertad de expresión. Este derecho encuentra especial correlato con el accionar de la prensa: "El público raciocinante, cada vez más amplio y crítico, encuentra su refuerzo natural en una prensa cada vez más crítica e independiente" (Monzón, 1985: 85).

Pers pectivas de la Comunicación - Vol. 13 - No2 - 2020 - pp. 73-103

Universidad de la Frontera - Chile 
Por su parte, Siebert (1984) habla de una "teoría libertaria de la prensa”, en la cual señala que la prensa, en el contexto liberal, debe su fundamento a una forma negativa de observar al Estado y al Poder, detentado sin contrapesos por monarquías absolutas en el marco del antiguo régimen. Para justificar este rechazo al Estado, se plantea que la censura de parte del Estado podría estar coartando la aparición de la verdad o, al menos, un germen de ella (Siebert, 1984: 46). En función de ponerle límites al Estado surge entonces la idea de la libertad de prensa, como derecho estrechamente relacionado con la libertad de expresión. Esto es, una prensa que se posicione en una vereda distinta al poder político, de tal forma que incluso es capaz de interpelarlo y controlarlo. Este es el origen de la idea de la prensa como cuarto poder, como vigía de los poderes establecidos (Siebert, 1984: 47). Salwen (1988) señala, a propósito de lo planteado por Siebert, que la prensa debería desempeñar una función de "perro guardián" y vigilar al gobierno por si comete indiscreciones o abusos de poder (Salwen, 1988: 132). A este rol independiente y controlador del poder, se suma un rol educativo de la prensa, pues cumple una importante función en la formación de un público informado y raciocinante. (Ruiz, 2003: 84; Siebert, 1984: 47).

En síntesis: del rol "independiente" que se le otorga a la prensa, surge la idea del cuarto poder, la idea del periodismo fiscalizador y la necesidad de que existan leyes de prensa que posibiliten el libre ejercicio de la profesión. Del rol que se le asigna a la prensa en torno a su participación en la gestación y desarrollo de una opinión pública, como guía, informador y educador de los ciudadanos, se desprenden conceptos como: objetividad, neutralidad, veracidad, formación de opinión. Estas ideas encuentran su fundamento precisamente en que, debido a las consideraciones expuestas anteriormente, se entiende a la prensa y al periodismo como un oficio necesario y valioso para el desarrollo correcto de un sistema político liberal.

\section{El mito liberal del periodismo}

Ahora bien, es posible verificar que estos valores asociados al periodismo moderno en el contexto de la Ilustración, tienen vigencia en lo discursivo en tiempos actuales. Esto se puede constatar al observar la gran cantidad de textos que se enseñan en numerosas escuelas de periodismo a modo de manuales, y que plantean la ética y el deber ser de los periodistas en épocas contemporáneas. 
Entre estos autores se destaca Gomis (1991), el cual plantea que el periodismo actúa como un mediador efectivo y objetivo entre la realidad y los públicos, señalando que esa "es su función social" (Gomis, 1991:175). Este autor, además, menciona que "lo que no pasa por los medios no pasa por ninguna parte, no deja constancia y no influye. Los medios son el lugar común de la acción pública. En esto consiste su mediación generalizada” (1991: 179). De igual forma, Taylor y Teel (1985), en un texto introductorio al periodismo, señalan que "nunca como entre los periodistas de ahora, la objetividad es un ideal. Después de todo, hemos alcanzado la jerarquía del cuarto poder. En la era de la información, somos los árbitros" (Taylor y Teel 1985: 112-113). Javier Darío Restrepo (2004), plantea que para el periodista: "La ética no es una condición ocasional, sino que debe acompañar siempre al periodismo como el zumbido al moscardón” (2004: 11). Por su parte, Kapúscinski (2003), plantea el deber ser de los periodistas en torno a imperativos éticos similares a los revisados con anterioridad. Sin embargo, este autor desarrolla la idea nostálgica de que el periodismo se ha ido alejando de esos valores normativos. Señala: "Normalmente el periodismo se hacía por ambición o por ideales, pero de repente se advirtió que la noticia era negocio, que permitía ganar dinero pronto y en grandes cantidades" (Kapúscinski, 2003: 23). Además, el Código deontológico europeo de la profesión periodística (Núñez, 1993), señala que los productos del periodismo "revisten una gran importancia para el desarrollo de la personalidad de los ciudadanos así como para la evolución de la sociedad y la vida democrática” (1993: 1). Por su parte, el Código de Ética del Colegio de Periodistas de Chile, señala que "quienes ejercen el periodismo son importantes agentes socializadores que tienen influencia en la formación devalores, creencias, hábitos, opinión y conductas de los distintos estamentos de la sociedad" (2015: 1). Además, respecto del deber ser del periodista, el código de ética señala que "los periodistas están al servicio de la sociedad, los principios democráticos y los Derechos Humanos. En su quehacer profesional, el periodista se regirá por la veracidad como principio, entendida como la entrega de información responsable de los hechos" (2015: 2).

En el mismo sentido que lo revisado en el apartado sobre el mito moderno, parece ser que un relato entablado en torno a los valores mencionados anteriormente es el que permite a los periodistas entender su lugar en el mundo, su ubicación en el contexto. Siguiendo lo planteado por Lévi-Strauss (1995), Schoenbach (2001) y Fernández (1996), es posible hallar los rastros de una estructura binaria, de una antinomia, que propone de manera enfrentada una división entre las buenas prácticas y las malas prácticas; entre los periodistas de verdad, 
y los periodistas de mentira; entre la objetividad y la subjetividad; entre la información y la opinión; entre la independencia y la parcialidad; en definitiva: entre el bien y el mal, periodísticamente hablando. Todas estas categorías constituyen un relato que auxilia a los periodistas a la hora de justificar el desarrollo de su propia actividad profesional. Ahora bien, si seguimos lo planteado por Barthes (1999), surge la idea de que el mito liberal del periodismo, opera naturalizando ciertos sentidos. De esta forma, al dar por sentado que un buen periodista siem pre cumplirá con un catálogo de valores, se está naturalizando que las obras de estos profesionales - sus noticias, sus reportajes, sus crónicas- se encuentran o deberían encontrarse imbuidas de las características que singularizan al ejercicio del periodismo: objetividad, independencia, rol fiscalizador, entre otros. De la misma forma, los productos periodísticos - como diarios y revistas - también resultan imbuidos del relato mítico del periodismo: en tanto se trata de objetos emanados del resultado de un proceder periodístico, se encuentran dotados de objetividad, independencia, información verídica, entre otros conceptos.

Ahora bien, es posible señalar que estas prerrogativas corresponden más al plano discursivo que al práctico. Esta afirmación puede encontrarse en el trabajo de numerosos autores que observan de manera crítica el ejercicio del periodismo.

Salinas y Stange (2009, 2014), señalan al respecto que las rutinas que deben realizar los periodistas en el ejercicio de su trabajo han desembocado en una progresiva burocratización que no dice relación con los valores que estos esgrimen. Algunos autores señalan que la objetividad y la neutralidad, entre otros valores del periodismo, constituyen un ideal virtualmente imposible de alcanzar, de tal forma que su búsqueda resulta un esfuerzo infructuoso y motivado por otros intereses (Chillón, 2009; Donsbach y Klett, 2014; Parra Pujante, 2012; San Martín, 2008; Tuchman, 1983, 1999).

Sumado a esto, hay algunos autores que plantean la existencia de un cambio contextual para el periodismo, el cual ha debido acomodarse progresivamente a un contexto neoliberal de prensa. Guerrero y Márquez (2014), plantean la existencia de modelos liberales capturados respecto de los medios de comunicación, dando cuenta de la progresiva acumulación de poder y formación de monopolios en torno a medios comunicacionales. Salinas, Santa Cruz y Stange (2018) plantean que, en el marco del modelo neoliberal, "se produce la reducción de toda estrategia comunicacional, incluyendo las de propaganda política, las periodísticas 
y las de desarrollo social o bien público, a estrategias publicitarias de mercado" (2018: 187). Otano y Sunkel (2005) se preguntan por qué, una vez llegada la democracia en Chile, se cerraron medios que daban cuenta de una sana pluralidad, y por qué no se han visto avances en términos del derecho a la libertad de expresión. Los autores llegan a la conclusión de que son elementos propios de la cultura del periodismo los que impidieron esta apertura.

De esta forma, es posible observar que la práctica periodística no se encuentra de manera necesaria en correlato con respecto al relato mítico sobre el cual están construidos los valores y justificaciones de dicha profesión.

¿Nuevos mitos sobre periodismo? El periodista como comunicador estratégico A propósito de esta falta de correlato entre el mito liberal del periodismo y el ejercicio práctico de la profesión, surgen nuevas discursividades sobre el periodismo, asociadas a nuevas aplicaciones prácticas en las cuales los periodistas desarrollan su trabajo. Al respecto, una serie de autores plantean que los periodistas egresados de escuelas de periodismo están cada vez más ejerciendo su labor profesional como comunicadores estratégicos o corporativos en organizaciones y empresas (Contreras, 2006; Corrales, 2006; Mellado, 2009a).

A propósito de lo mismo, hay abundante literatura que se refiere a los periodistas dedicados a este tipo de labores, las cuales dicen relación con organizar y planificar de manera estratégica las comunicaciones de empresas y organizaciones (Almansa, 2004, 2005; Arras, Jáquez y Fierro, 2008; Contreras, 2006; Pérez, 2008; Rogers y Agarwala-Rogers, 1980; Salas, 2011). Estos autores, de distintas formas, plantean la importancia que reviste la comunicación para estas organizaciones y empresas, de tal forma que debe ser tratada de manera estratégica. Generalmente, el propósito de esta visión estratégica dice relación con conseguir o mantener una buena imagen frente a determinadas audiencias; además de fortalecer una adecuada comunicación con el entorno de la organización "donde el emisor decide y preelabora la comunicación de cara a unos objetivos, de acuerdo con un contexto o unas tendencias, para las que hay que tener en cuenta las posibles decisiones/reacciones de los públicos objetivos, tanto internos como externos" (Pérez, 2008: 236). Se plantea, a partir de estas ideas, que existe una "nueva comunicación”, la cual "siempre debe ser estratégica, la investigación es cuando se escucha a nuestros públicos e instituciones, y la base de nuestra estrategia o planes en comunicación, que afectan a toda la organización. Esta se convierte 
en el soporte de nuestra gestión gerencial, que busca el entendimiento mutuo de una organización con sus diversos públicos, tanto internos como externos” (Salas, 2011: 243).

Es precisamente a propósito de estos discursos, que sostienen la idea de que el periodista puede y, eventualmente, debe dedicarse a estas nuevas labores, que pretendemos ahondar en la investigación que exponemos a continuación.

\section{Metodología}

El objeto de estudio que observaremos en esta investigación consiste en los elementos que plantean al periodista como comunicador estratégico presentes en el discurso de académicos que imparten asignaturas sobre Comunicación Estratégica y Organizacional en escuelas de periodismo de la Región Metropolitana de Chile. Como señalamos en la introducción, la hipótesis de trabajo consiste en señalar que el mito liberal del periodismo carece de suficiencia para explicar completamente el rol de los periodistas en el contexto contemporáneo. De esta forma, los discursos que plantean al periodista como un especialista en comunicación estratégica, operan como un nuevo mito que explica el rol de los periodistas en el contexto actual.

Desde esta hipótesis podemos desagregar dos variables para observar la verificación o refutación de esta hipótesis.

1. Variable dependiente: La suficiencia o rendimiento del mito liberal del periodismo para explicar el rol de los periodistas en el contexto contemporáneo.

2. Variable independiente: La verificación de que los discursos que plantean al perio dista como comunicador estratégico constituyen un relato mítico con poder explicativo sobre el rol de los periodistas en el contexto contemporáneo.

Verificada la variable independiente, constataremos que el mito liberal del periodismo no es totalmente suficiente para explicar el rol de los periodistas en el contexto contemporáneo, de tal forma que se encuentra permeado por otros relatos míticos con poder explicativo de la profesión. Luego de esto se analizará de qué forma se encuentran tensionados ambos relatos, es decir: observar los principales conceptos de este eventual nuevo mito y sus similitudes o desavenencias con las formas tradicionales de ver el periodismo; teniendo en cuenta, además, que se trata de formas de entender la profesión que conviven al mismo tiempo. 
Para observar la existencia de un mito en los discursos que plantean al periodista como comunicador estratégico, retomaremos lo desarrollado en el marco teórico a propósito de lo que denominamos mito moderno. En el desarrollo de tales conceptualizaciones, rescatamos lo propuesto por Barthes (1999) y Lévi-Strauss (1995), desde los cuales se extraen ciertas características propias de un habla mítica, y así formular indicadores que permitan verificar la existencia de un mito en un discurso. La presencia de estos indicadores en los discursos que analizaremos nos señalará la presencia de un habla mítica, lo cual nos llevará a verificar la variable independiente y, por consecuencia, la hipótesis de trabajo.

Podemos identificar tres dimensiones presentes en el concepto de mito las cuales debemos hallar en los discursos que analizaremos para afirmar la presencia de un habla mítica. A saber: 1) el mito es un habla, una discursividad sobre un objeto; 2) el mito contiene antinomias y oposición binaria de categorías, las cuales explican y dotan de sentido al individuo en un contexto; 3) el mito es un habla despolitizada por cuanto desliza y naturaliza un sentido descontextualizado, desconectado de su historia. De esta forma, los indicadores según los cuales observaremos la presencia de un mito son:

1. Para la primera dimensión: La presencia de una excesiva discursividad en torno a ideas y criterios puntuales sobre el deber ser de los periodistas como comunicadores estratégicos. La reiteración de formulaciones normativas sobre el periodista como comunicador estratégico develará las predicaciones fundamentales respecto del objeto, en este caso, el rol de los periodistas como comunicadores estratégicos.

2. Para la segunda dimensión: La presencia de estructuras antinómicas o binarias en los discursos que entreguen potencial explicativo y que sitúan al periodista en un contexto específico, entregándole justificación y explicando su rol en el contexto contemporáneo.

3. Para la tercera dimensión: La presencia de elementos naturalizados, no justificados, o dados por hecho en el discurso que presenta a los periodistas como comunicadores estratégicos. En la medida que se encuentren naturalizadas ciertas habilidades, roles o características que deben tener los periodistas o sus productos, en los discursos sobre comunicación estratégica, se está en presencia de un habla mítica.

Teniendo en cuenta que el periodismo constituye una práctica que tradicionalmente era entendida como un oficio, pero que durante el siglo XX progresivamente se fue profesionalizando e integrando a programas universitarios de educación (Cabalín y Lagos, 
2012: 160; Mellado, 2009b: 195-196), entendemos a la universidad y sus escuelas de periodismo como la institución en que se producen y reproducen con mayor fuerza los discursos sobre el ejercicio del periodismo en cuanto profesión.

A propósito de este argumento, según datos del Ministerio de Educación de Chile (MINEDUC), en la Región Metropolitana hay 17 planteles universitarios que ofrecen la carrera de periodismo. Entre estas universidades se cuentan dos que son consideradas estatales: la Universidad de Chile y la Universidad de Santiago de Chile; y 15, las cuales son consideradas privadas4. En una rápida revisión, se puede observar que la totalidad de ellas ofrecen en sus mallas curriculares de periodismo, la posibilidad de realizar cursos sobre comunicación estratégica/organizacional/corporativa. De todas ellas, 16 ofrecen ramos de comunicación estratégica en sus mallas de manera obligatoria, y una sola de ellas (la Pontificia Universidad Católica de Chile) provee ramos de estas materias mediante "Optativos de Profundización Periodística", los cuales son asignaturas electivas. En varios de estos programas de educación superior se pueden observar hasta cuatro ramos obligatorios relacionados con materias sobre comunicación estratégica 5. Esto nos permite observar la importancia y espacio que han ganado estas materias en el desarrollo curricular de las universidades chilenas que imparten periodismo.

Para todo lo anterior, se decidió analizar un corpus constituido por académicos que se desempeñen laboralmente en universidades reconocidas por el MINEDUC en la Región Metropolitana de Chile, los cuales ejercen docencia en escuelas de periodismo impartiendo clases de comunicación estratégica/organizacional/corporativa. Los discursos de estos

\footnotetext{
4 La información sobre aquellas universidades reconocidas por el Ministerio de Educación de Chile, que ofrecen carreras de per iodismo, puede consultarse en el sitio web www.mifuturo.cl. Este sitio depende del Ministerio de Educación y su principal ca racterística es que provee de información, fundamentalmente, a aquellas personas que desean ingresar a cursar carreras de educación superior en universidades chilenas. La información expuesta aquí fue consultada el 29 de agosto de 2019 en el sitio w eb mencionado.

5 Los datos que se presentan sobre la constitución de estas mallas curriculares fueron consultados en los sitios web de cada u na de estas instituciones de edu cación su perior a la fecha del 29 de agosto de 2019. Todos estos programas universitarios (los 17 a ntes m encionados) proveen acceso público a sus respectivas mallas curriculares. Los sitios consultados fueron: www.umayor.cl; www.academia.cl; www.ust.cl; www.udla.cl; www.unab.cl; www.uai.cl; www.uniacc.cl; www.uft.cl; www.uandes.cl; www.udp.cl; www.udd.cl; www.ucentral.cl; www.ubo.cl; www.uahurtado.cl; www.uchile.cl; www.uc.cl; www.usach.cl
} 
académicos fueron recolectados mediante entrevistas semiestructuradas, entendiendo que tales entrevistas constituyen conversaciones o "textos negociados" con el entrevistado (Fontana y Frey, 2017: 179). Se plantearon las entrevistas a propósito de tópicos importantes sobre los vínculos entre periodismo y la comunicación estratégica como disciplina, de manera que en esos discursos se pudiera detectar la aparición de elementos propios de un mito.

El criterio utilizado para considerar suficiente la producción de datos atingente a la investigación consiste principalmente en la observación de aspectos y características recurrentes y coincidentes en los discursos de estos académicos. Una vez observadas suficientes recurrencias en torno a determinados aspectos constitutivos de mito, entonces se considerará un corpus suficiente de académicos para comenzar a realizar el análisis. Lo anterior se sigue de que esta investigación pretende proceder mediante un método cualitativo de investigación que permita producir y analizar datos en función de su significatividad $-\mathrm{y}$ no su cantidad - para la investigación, poniendo relevancia en los discursos y en el análisis del sentido que aparezca en ellos. Además de esto, esta investigación tiene un enfoque exploratorio pues, si bien plantea una relación directa entre dos variables, se trata de "explorar algo poco investigado o desconocido" (Hernández, Fernández y Baptista, 1991: 70). Además, se trata de una investigación empírica, pues pretendemos obtener los discursos observando un fenómeno de la realidad mediante un trabajo de campo.

Los resultados fueron analizados mediante la técnica del Análisis del Discurso (AD), puesto que las materialidades que nos entregan estas conversaciones nos serán útiles en la medida que podamos establecer o conocer un trasfondo contextual desde el cual se produce el discurso particular. Van Dijk señala la importancia de esta relación entre lo particular y lo contextual: "la disciplina estudios del discurso debería tratar tanto de las propiedades del texto como las de la conversación, y de lo que se denomina el contexto, es decir, las otras características de la situación social” (Van Dijk, 2000: 24). Este contexto -o situación social- al cual buscamos acceder mediante estas conversaciones no es otro que la eventualidad de la presencia de un habla mítica compartida por una comunidad. En el mismo sentido, Manzano (2005) señala que en un discurso se pueden identificar dos dimensiones principales: una amplia y una concreta. En este sentido, existe un "discurso concreto" que consiste en el habla particular de un agente que dice algo, pero además, existe un "discurso ampliado", al cual el discurso particular hace referencia (Manzano, 2005: 2). 
Nuevamente; ese discurso ampliado que buscamos detectar es la presencia de un habla mítica compartida por estos académicos sobre el periodismo y la comunicación estratégica.

\section{Resultados}

Los entrevistados dan cuenta de una recurrencia discursiva respecto de ciertos elementos. Esto es de gran importancia pues, como señalamos en la construcción teórica de esta investigación, un mito es un relato compartido por una comunidad. Una vez entrevistado el cuarto académico hemos encontrado suficientes recurrencias como para detenernos a extraer resultados y realizar el análisis. A continuación, se presentan los principales resultados de manera simplificada en categorías, para así facilitar su comprensión, y la extracción de conclusiones atingentes a la investigación.

\section{a. La idea antinómica del pasado ineficiente y la necesidad presente de la eficiencia: profesionalización, planificación, estrategia.}

Se observa de manera recurrente en los discursos de estos académicos que, cuando se refieren a la comunicación estratégica apuntan a la necesidad de tener al fenómeno de la comunicación bajo control. Los entrevistados señalan recurrentemente que en un tiempo pasado no se aprovechaba todo el potencial de la comunicación, de tal forma que la comunicación estratégica plantea una forma profesional, estratégica y, eventualmente, científica de observar el fenómeno en la actualidad. Se propone que la comunicación estratégica en organizaciones viene a poner orden allí donde imperaba el desaprovechamiento. Es de esperar que esto esté vinculado con la idea de que la comunicación, de alguna forma, pasa a considerarse un insumo, la cual incluso es capaz de generar beneficios económicos a las organizaciones. Algunas citas ilustrativas al respecto son: "El foco sobre las organizaciones tiene que ver con la idea de hacerlas más eficientes, de mejorar todos los procedimientos en su interior (...)”; o bien: “(...) La materia principal, primero, es tener un pensamiento estratégico. Y el pensamiento estratégico tiene siempre dos puntos. El dónde estamos ahora, que requiere un buen diagnóstico, investigación (...)”. Además, a propósito de este tránsito entre un pasado y un futuro: “(...) la comunicación era entendida casi como salgamos en el diario. Me acuerdo super bien de la frase espantosa que todavía se usa: "hagamos ruido". Pero afortunadamente ese hacer ruido, fue derivando en: 'digamos lo que queremos y lo que tenemos que decir'. Eso es lo más importante, más que una necesidad imperiosa de comunicar, hay una necesidad hoy día 
de comunicar bien y de comunicar muy bien a quién: las organizaciones tienen que elegir y tienen que definir a quienes le comunican (...)”.

\section{b. La antinomia del pasado inconducente y la readecuación a los nuevos tiempos: La migración del periodismo por necesidad}

Esta idea también dice relación con una antinomia temporal. En este caso, se observan discursos similares a aquellos en que se justifica una migración: la necesidad material, necesidad de mejores condiciones, la inconducencia del pasado, la insuficiencia de puestos de trabajo, la búsqueda de un lugar mejor, entre otros elementos. En este sentido, se plantea que el periodista debe aprovechar sus habilidades comunicativas, las que ha desarrollado a lo largo de una formación periodística tradicional, para ponerlas al servicio de aquellas actividades que involucran un contexto materialmente más favorable dado que el periodismo tradicional ya no va a cumplir con las necesidades de los nuevos egresados de periodismo. Citas ilustrativas al respecto son: “(...) lo primero es la razón del mercado, o sea, no hay puestos de trabajo en medios, por lo tanto, lo que uno hace o lo que se havenido haciendo en las escuelas es abrirse a una suerte de adaptación a lo que está sucediendo en nuestra realidad (...)”; además: “(...) Yo creo que también hay una necesidad del mercado. En el sentido de que con la cantidad de Escuelas de Periodismo que habían, los medios de comunicación se empezaron a achicar y había que buscar nuevos nichos de trabajo”.

\section{c. La antinomia del actual público complejo versus el público simple del pasado}

También se observa una antinomia temporal respecto a los públicos a los que va dirigida la comunicación. En este sentido, se menciona reiteradamente que la situación del público actual, de las audiencias o receptores, es radicalmente más compleja que en el pasado. Se habla sobre públicos más educados, más conectados, más exigentes y más particularizados. En estos discursos aparece la noción de las audiencias segmentadas, y de cómo la comunicación estratégica permite considerar numerosos involucrados y receptores del mensaje que se pretende comunicar. Mediante la idea de la planificación y la estrategia se busca conectar con estos públicos complejos de manera más adecuada y eficiente.

Algunas citas que grafican de buena manera esta situación son, por ejemplo: “(...) a medida que vayamos avanzando en el tiempo, a propósito de la opinión pública, en lo críticos que están, en las redes sociales; va a ser más importante que esas organizaciones tengan una

Pers pectivas de la Comunicación - Vol. 13 - No2 - 2020 - pp. 73-103

Universidad de la Frontera - Chile 
idea o una imagen coherente dentro de la opinión pública”; además “(...) Sile hablas a todos no le hablas a nadie. Entonces tienes que segmentar... un alumno no tiene por qué tener la mismaimpresión que un profesor, o que un funcionario. Pertenecen al mismo espacio, pero no a la misma comunidad de intereses (...)”.

\section{d. La sobrediscursividad de lo económico}

Se observa de manera transversal la presencia de numerosos términos provenientes desde disciplinas relacionadas con la economía y los negocios. De manera naturalizada se utiliza la voz "clientes" para referirse a la organización a la que se asesora; también se observa que en ocasiones se utiliza de manera directa la voz "empresas", o "negocio" en vez de organizaciones, que es el término más frecuente y formal. Se utilizan una serie de conceptos venidos desde el marketing y la ingeniería comercial. Además de esto, es interesante constatar cómo la comunicación se observa desde una perspectiva de insumo que crea valor económico. En ese sentido, la comunicación es vista como un factor que permitirá un mayor beneficio económico para la organización.

\section{e. La refacción de elementos del periodismo tradicional}

Se observa de manera muy frecuente la idea de reciclar o refaccionar elementos propios del periodismo tradicional, es decir, el periodismo de medios, para ponerlos al servicio de estas nuevas prácticas orientadas a las empresas. La figura propia del periodismo tradicional, que es refaccionada con mayor frecuencia para ser aplicada a funciones de Comunicación Estratégica, es la del "reporteo". El reporteo, entendido como el tipo de investigación que realiza habitualmente un periodista de medios, es puesto al servicio de realizar diagnósticos para las empresas, conocer los involucrados en las comunicaciones dela organización, o para comprender la naturaleza de la situación comunicacional que se enfrenta. Se señala de manera repetitiva que esta característica pone a los periodistas en un sitial de privilegio para ocupar puestos de trabajo en organizaciones.

Sumado al reporteo, se encuentra la idea de que el periodista maneja bien la producción de relatos. En tanto que las organizaciones o empresas necesitan comunicar de manera clara y creativa a sus clientes, esta habilidad periodística resulta muy útil, pues puede articular los mensajes que necesita realizar la organización hacia sus públicos. 
También se observa una refacción de la idea del "olfato periodístico", por cuanto el periodista es capaz de observar lo que ocurre en los públicos, o en el "fenómeno comunicacional".

Algunas citas significativas al respecto son: (...) Lo que podemos observar es el trabajo de periodistas en organizaciones incluso realizando labores periodísticas. O sea, de reporteo. No es solamente pensar que la comunicación estratégica se ejerce desde la planificación, sino que también muchas veces hay organizaciones que tienen verdaderos medios en su interior (...)”. Además: “la capacidad de decir: esto es más o es menos importante, vamos a estructurar los mensajes de tal manera que sean comprensibles a cada una de nuestras audiencias. Eso lo puede hacer un periodista (...). Un publicista no va a tener la capacidad que tenemos nosotros de desarrollar en cuatro líneas un mensaje que tenga contenido". Además, “(...) por ningún motivo hay que olvidarse del periodismo, porque eso es lo que nos da el valor agregado. El saber reportear, entender, análisis, contextos, contenidos (...)".

\section{f. La noción de "comunicación" como elemento que conecta a los periodistas con la comunicación estratégica}

Es recurrente la mención que se hace del término "comunicación" como aquel que permite a los periodistas dedicarse a la comunicación estratégica. En este sentido, en la medida que los periodistas se presentan como profesionales expertos en "comunicación", son sujetos aptos para desempeñar labores como comunicadores estratégicos en las organizaciones o empresas. Esto se debería a que, como mencionamos antes, la comunicación pasa a ser vista como un insumo valioso para las empresas. Más allá de si los periodistas realizan noticias, crónicas y reportajes, el hecho de que ellos - eventualmente-comprendan el fenómeno comunicacional y sus factores permite decir que pueden manejar los aspectos comunicacionales de una organización. Esto implica que el periodista, cada vez más, se plantea como un "comunicador" más que como un "periodista”.

Algunas citas ilustrativas al respecto son: “(...) todas las organizaciones necesitan comunicación: ministerios, el Ejército, todas las ramas de las Fuerzas Armadas, los propios medios muchas veces necesitan comunicación interna, cosa que no muchas veces se preocupan de darse; las universidades, los colegios. ¿Dónde no?”; además: “(...) esto se los digo mucho a mis alumnos, no se trata de que sea una Escuela de Periodismo, o una Escuela de Relaciones Públicas, sino que se trata de formar Escuelas de Comunicación

Pers pectivas de la Comunicación - Vol. 13 - No2 - 2020 - pp. 73-103

Universidad de la Frontera - Chile 
Social (...) entonces, tener una Licenciatura en Comunicación Social, entender lo que es la comunicación desde lo social, te va a permitir trabajar en medios y en empresas (...)”.

\section{g. La incertidumbre de si la comunicación estratégica es o no es una forma de periodismo}

Durantelas entrevistas aparece en numerosas situaciones elem entos que llevan a pensar que los académicos no piensan que la Comunicación Estratégica sea lo mismo que el Periodismo. Algunos entrevistados señalan que el periodismo y la comunicación estratégica son lo mismo, pues son “dos caras del mismofenómeno: la comunicación”. Sin embargo, no existe un acuerdo total respecto de si son lo mismo. En numerosas ocasiones se hace alusión a "los comunicadores" por contraposición a "los periodistas". De la misma forma, se habla de periodistas que se dedican a "periodismo" y periodistas que se dedican a "comunicaciones". Es interesante constatar estas formas de hablar naturalizadas que, lo que dejan claro, es más bien que no hay demasiada certidumbreal respecto. Ni siquiera los académicos que enseñan estas materias están tan seguros de si la comunicación estratégica es o no es periodismo

\section{Análisis y conclusiones}

Como podemos observar en los resultados de estas entrevistas, existen elementos propios de un mito, según la conceptualización que hemos descrito en el marco teórico sobre mito moderno. Ahora bien, si el mito liberal del periodismo se resume en un relato que, debido a una serie de razonamientos propios de la modernidad, plantea como eje central una serie de valores: objetividad, independencia, fiscalización del poder, cuarto poder, entre otros; ¿Cuáles son los elementos que articulan este eventual nuevo mito sobre el periodista como comunicador estratégico?

1. Las primeras tres de las categorías revisadas son antinomias temporales que plantean una dicotomía entre lo pasado/lo nuevo. Se observa: la necesidad de eficientizar, profesionalizar, y hacer un mejor producto con la comunicación; la migración por necesidad desde un contexto ya acabado para acercarse a contextos que deparen mejores situaciones materiales para los periodistas; y el advenimiento de un tipo de público o escenario de audiencias cada vez más complejo y difícil de asir. El elemento que comunica estas tres antinomias dice relación con la necesidad de adecuación de los periodistas a nuevos tiempos, porque si no lo hacen sufrirán las consecuencias de manera material. En este sentido, el mito del periodista 
como comunicador estratégico, tiene un primer elemento: "la renovación", o bien "estar a la altura de los nuevos tiempos”.

2. Esta renovación dice relación con ponerse a tono con un contexto altamente competitivo, en que lo económico y lo comercial dominan el escenario. Esto explica que esta renovación se dé al alero de discursividades cercanas al mundo de la economía y los negocios: es necesario volver al periodismo una actividad rentable en el sentido de los nuevos tiempos. En la época moderna, en la cual se desarrolla el periodismo liberal; la objetividad, la independencia y el rol fiscalizador, son atributos del periodista que tienen un valor incalculable. Ese valor bien se podía traducir en reputación, fama, dinero, relaciones sociales, bienes, entre otras cosas. En tiempos contemporáneos, el valor que se le adjudica a una actividad, aparentemente, está relacionado con la rentabilidad económica que esta actividad suscita. Si una actividad no genera una rentabilidad suficiente, entonces podríamos señalar que es menos valorada por su contexto. En este caso, el periodismo entendido como el periodismo tradicional, carece de valor en contexto contemporáneo. Se suma otro elemento al mito del periodista como comunicador estratégico, consistente en dotar de valor al ejercicio actual del periodismo: "Hay que darle valor a nuestra actividad".

3. A propósito de lo que mencionamos como "refacción de elementos del periodismo tradicional", los discursos observados plantean elementos tradicionales del periodismo liberal en función de un nuevo escenario. El reporteo, la capacidad de relatar o crear contenidos, en tanto que habilidades desarrolladas por los periodistas, son reutilizadas, recicladas, para así poder servir a nuevos propósitos que nada tienen que ver con aquellos para los que tradicionalmente fueron concebidos. Es interesante, para este caso, observar cómo opera la idea de naturalización en el lenguaje desarrollada por Barthes sobre los mitos. Recordemos que según este autor los mitos naturalizan sentidos, debido a que utilizan un significante ya dotado de sentido como si estuviera vacío. En este caso, se trata de las nociones de reporteo, capacidad de relato y habilidades comunicativas; las cuales son vaciadas de su sentido original periodístico, vehiculizando un nuevo sentido orientado a los objetivos de las organizaciones o empresas. A modo de ejemplo: el reporteo es útil para realizar un buen diagnóstico de la situación comunicacional de la empresa u organización. Se habla de reporteo en las organizaciones y, precisamente porque se trata de reporteo, se desliza la idea de que aquello que resulte de este reporteo constituirá un producto periodístico. Lo mismo sucede con la capacidad de relato o habilidad comunicativa. Bien 
puede sostenerse que esta naturalización es una de las razones que permite que se enseñe Comunicación Estratégica/Organizacional/Corporativa en las escuelas de periodismo. El uso de términos periodísticos, reacondicionados para el campo de las organizaciones y empresas, es el que permite la vinculación de los periodistas con estas nuevas materias. Sin embargo, hay que tener especial atención respecto de esta vehiculización de sentido pues, de alguna forma, indica cierta permanencia del mito liberal del periodismo. No de sus atributos principales -objetividad, independencia, fiscalización al poder- pero sí de elementos que se siguen de esos atributos, como son el reporteo, la habilidad para crear relatos, entre otros.

De esta manera, se desprende un nuevo elemento del mito del periodista como comunicador estratégico: "las habilidades tradicionales del periodismo tradicional son útiles a las empresas u organizaciones”.

4. Además de esto, planteamos la idea de la centralidad de la noción de "comunicación” en los discursos analizados. El concepto “comunicación” es el eslabón quevincula al periodismo tradicional con la comunicación estratégica. En el contexto moderno, en el que se desarrolla el mito liberal del periodismo, se plantea que los periodistas, a través de sus productos periodísticos - noticias, reportajes, etc.- comunican contenidos que constituyen parte importante del desarrollo de una opinión pública. Esto lo consiguen a través de los medios de comunicación. Es decir, los periodistas son profesionales que tradicionalmente se han encargado de comunicar sus contenidos periodísticos a una audiencia masiva. En el contexto contemporáneo, a propósito de las organizaciones y empresas, se observa la comunicación como un insumo que proveerá de beneficios materiales a la empresa, de tal forma que constituye un elemento que conviene controlar y manejar de manera óptima.

En ambos casos se habla de comunicación, aunque se trata de formas de entenderla diametralmente opuestas. Es el uso del término "comunicación”, al cual los periodistas se sienten relacionados por haber trabajado desde siempre en medios de comunicación, el que permite que estos periodistas puedan justificar su trabajo planificando la comunicación estratégica de las organizacionesy empresas. Esto supone un giro de parte de los periodistas, desde considerar como objeto propio de su oficio: la producción de contenido periodístico; a la comunicación en todas sus formas. El periodista gira desde lo exclusivamente periodístico hacia la comunicación o lo comunicacional, como noción más amplia. 
Considerando el análisis realizado sobre estos discursos, podría sostenerse que el siguiente enunciado contiene los elementos que justifican al periodista, de manera mítica, como comunicador estratégico: el mito del periodista como comunicador estratégico.

El periodista es un profesional de la comunicación, renovado y a la altura de los tiempos, que valoriza su actividad en este contexto contemporáneo, pues la comunicación es muy importante para generar beneficios en empresas y organizaciones.

Esta investigación corrobora de manera parcial la hipótesis de trabajo, la cual es: "El mito liberal del periodismo carece de rendimiento para explicar completamente el rol de los periodistas en contexto contemporáneo, de tal forma que los discursos que plantean al periodista como comunicador estratégico operan como un nuevo relato mítico que explica el rol del periodista en el contexto actual, tensionando los conceptos del mito liberal del periodismo".

Se plantea la corroboración parcial de esta hipótesis, puesto que si bien existen elementos míticos en los discursos analizados que suponen una superación de la forma liberal y tradicional de observar al periodismo, de igual form a se utilizan aspectos secundarios de este mito liberal del periodismo - reporteo, investigación, capacidad de producir relatos y contenidos- para justificar el ejercicio de la profesión en la comunicación estratégica de organizaciones. Es decir, el periodismo moderno se supera, pero de igual forma se instrumentalizan ciertos aspectos de aquella forma moderna de entender el periodismo para así conseguir enganchar con las nuevas formas de concebir el periodismo: la comunicación estratégica en organizaciones. Deigual forma, se consigue sustentar la idea de que las formas tradicionales de observar el periodismo carecen de suficiencia absoluta para explicar la labor de los periodistas en la actualidad. Estas visiones tradicionales sobre el periodismo se encuentran permeadas por relatos con poder explicativo más acordes a las prácticas y contextos actuales. Para este caso, aquel que señala al periodista como comunicador estratégico.

Cabe señalar que la constatación de este mito sobre el periodista como comunicador estratégico efectivamente constituye un relato que permite a los periodistas justificarse en el ejercicio de estos nuevos roles: es un mito pues permite a los miembros de una comunidad

Pers pectivas de la Comunicación - Vol. 13 - No2 - 2020 - pp. 73-103

Universidad de la Frontera - Chile 
situarse y entenderse en un contexto. La razón principal de la necesidad de este mito es la incertidumbre que tienen incluso los académicos entrevistados a la hora de decir si la comunicación estratégica, como aplicación a la que se dedican los periodistas, es periodismo o no. Si los propios académicos que enseñan comunicación estratégica tienen algunas dudas sobre si lo que enseñan es periodismo o no, entonces parece lógico que constantemente se esté tratando de justificar que estas materias se enseñen en una Escuela de Periodismo.

En otras palabras, no se tiene absolutamente claro el por qué los periodistas son los profesionales idóneos para realizar las tareas propias de la comunicación estratégica de una organización, sin embargo, como es un territorio relativamente ganado por los periodistas, estos se empeñan - discursivamente- en justificarlo. No es de extrañar que estos discursos justificadores tengan como desembocadura la conformación de un relato mítico en los términos que lo hemos señalado durante esta investigación.

Esta investigación, además, toca de manera tangencial elementos que podrían ser tratados de manera más completa. Por ejemplo, la idea de que la noción de estrategia viene a intentar solucionar el surgimiento de audiencias o públicos segmentados (Salinas, et al. 2018). De la misma forma, es interesante observar cómo las propias organizaciones y empresas se constituyen como eventuales medios de comunicación, en tanto que son capaces de instalar mensajes en esta - ¿nueva? - opinión o esfera pública de manera eficiente. Realizan estas tareas comunicacionales con una eficacia, probablemente, mayor que la de los mismos medios de comunicación en la actualidad. Hay una correspondencia, entonces, entre una atomización o supersegmentación de las audiencias, con una atomización o supersegmentación en los “emisores”, puesto que las organizaciones y empresas, a través de sus propias herramientas comunicativas, constituyen verdaderos medios de comunicación supersegmentados.

Además de esto, es interesante observar o, más bien, intentar adivinar cuál será el futuro del periodismo en este problemático escenario. Es de esperar que los elementos descritos en el mito sobre el periodista como comunicador estratégico sean utilizados ampliamente para seguir buscando la introducción de los periodistas en materias sobre comunicación estratégica en organizaciones. A partir de esta reflexión, ¿cómo serán las escuelas de periodismo en el futuro? ¿Las habrá? O quizás, como señaló un entrevistado, dejarán de existir para dar paso a Escuelas de Comunicación Social, que sean capaces de englobar una 
gama más amplia de actividades. Es de esperar que, entre estas nuevas áreas desarrolladas por las escuelas de periodismo, se encuentren, cada vez más, aquellas que entreguen la posibilidad de que el periodista pueda llegar a las empresas, a sus gerencias y a sus directorios. 


\section{Referencias bibliográficas}

ALMANSA, A. (2004): Historia de los gabinetes de comunicación en España. Revista Historia y Comunicación Social, 9, pp. 5-21.

(2005): Relaciones públicas y gabinetes de comunicación. Anàlisi: quaderns de comunicació i cultura, (32), pp. 117-132.

ARRAS, A., JÁQUEZ, J. y FIERRO, L. (2008): Comunicación y cambio organizacional. Revista Latina de Comunicación Social, 11(63), pp. 418-134.

BARTHES, R. (1999): Mitologías. Ciudad de México: Siglo XXI.

BURRIDGE, K. (1968): Lévi-Strauss and myth. En The structural study of myth and totemism. Londres: Tavistock.

CABALÍN, C. y LAGOS, C. (2012): Enseñanza del periodismo en Chile y globalización: temas y desafíos. Signo y Pensamiento (61), pp. 158-170

CHILLÓN-LORENZO, J. M. (2009): Crítica y objetividad contra dogmatismo: lecciones popperianas para el periodismo informativo. Estudios sobre el mensaje periodístico, vol. 15, pp. 157-173.

Colegio de Periodistas de Chile (2015): Código de Ética. Santiago de Chile

CONTRERAS, D. (2006): La gestión de crisis en la comunicación organizacional: el caso de Chile. Anàlisi: quaderns de comunicació i cultura, (34), pp. 305-313.

CORRALES, O.(2006): Situación laboral de los periodistas egresados de la U. Chile (19932003). Cuadernos de trabajo ICEI (3). Santiago de Chile: Instituto de la Comunicación e Imagen, Universidad de Chile.

DONSBACH, W. y KLETT, B. (2014): Objetividad subjetiva: cómo definen periodistas de cuatro países un término clave de su profesión. En RUIZ, F. (Comp.). Cómo entender al periodismo: selección de la obra de Wolfgang Donsbach. Buenos Aires: Konrad Adenauer Stiftung. 
FERNÁNDEZ, J. J. (1996): Los sentidos del mito. Análisis comparativo de las visiones de R. Barthes, C. Levi-Strauss y K. Burridge. Revista Murciana de Antropología (3). Pp. 9-20.

FONTANA, A. y FREY, J. (2017): La entrevista. De una posición neutral al compromiso político. Manual de investigación cualitativa Vol. IV. México: Gedisa.

GOMIS, L. (1991):Losmedios, mediadores generalizados. En: Teoría del periodismo. Cómo se forma el presente. Barcelona: Paidós.

GUERRERO, M y MÁRQUEZ, M. (2014): El modelo 'iberal capturado' de sistemas mediáticos, periodismo y comunicación en América Latina. Temas de Comunicación, (29), pp. 135-170.

HABERMAS, J. (1981): Historia y crítica de la opinión pública: la transformación estructural de la vida pública. Barcelona: Gustavo Gili.

HERNÁNDEZ, R., FERNANDEZ, C. y BAPTISTA, P. (1991): Metodología de la investigación. México: McGraw-Hill.

KAPUSCINSKI, R. (2003): Los cinco sentidos del periodista (estar, ver, oir, compartir, pensar). México: Fundación para un Nuevo Periodismo Iberoamericano-Fundación Proa.

LÉVI-STRAUSS, C. (1995): Antropología estructural. Trad. E. Verón. Barcelona: Paidós.

MANZANO, V. (2005): Introducción al análisis del discurso. Disponible en: http://personal.us.es/vmanzano/docencia/metodos/discurso.pdf

MELLADO, C. (2009a): Evolución del campo ocupacional y académico del periodista latinoamericano: lógicas de mercado y esquemasde formación. Red de Revistas Científicas de América Latina y el Caribe, España y Portugal, (25).

(2009b): Periodismo en Latinoamérica: Revisión históricay propuesta de un modelo

de análisis. Red de Revistas Científicas de América Latina y el Caribe, España y Portugal, (18). 
MONZÓN, C. (1985). Orígenes y primeras teorías sobre la opinión pública: El liberalismo y el marxismo. Revista de estudios políticos, (44), pp. 81-114.

(2006): Opinión pública, comunicación y política (2a. ed., Colección Biblioteca Universitaria). Madrid: Tecnos.

NÚÑEZ, M. (1993): Código Deontológico Europeo de la Profesión Periodística. Disponible en:

http://www.asociacionprensa.org/es/images/Codigo_Deontologico_Europeo_de_la_Prof esion_Periodistica.pdf

OTANO, R. y SUNKEL, G. (2005): Libertad de los periodistas en los medios. En: Mordazas de la Transición. Santiago de Chile: Programa para la Libertad de Expresión. pp. 29-51.

PARRA-PUJANTE, A. (2012): La lógica periodística en sentido epistemológico. Estudios sobre el mensaje periodístico, 18 (2), pp. 891-906.

PÉREZ, R. (2008): Estrategias de comunicación. Barcelona: Book Print Digital.

RESTREPO, J. D. (2004): El zumbido y el moscardón: taller y consultorio de ética periodística. pp. 17-43. México: Fondo de Cultura Económica.

RODRIGUEZ, R. F. (1998): La objetividad periodística, un mito persistente. Revista Latina de comunicación social, 2(5), pp. 1-6.

ROGERS, E. y AGARWALA-ROGERS, R. (1980): La Comunicación en las organizaciones. México: McGraw-Hill.

RUIZ, C. (2003): El republicanismo en las teorías de la prensa en Chile durante el siglo $X I X$. Derecho y Humanidades, (9).

SALAS, C. (2011): Estado del arte de la nueva comunicación estratégica en Iberoaméricay Colombia. Signo y Pensamiento, 31(59).

SALINAS, C., SANTA CRUZ, E. y STANGE, H. (2018): Prensa y sociedad. Estrategias y modelos periodísticos en el Chile neoliberal. Transformaciones de la esfera pública en el 
Chile neoliberal. Luchas sociales, espacio público y pluralismo informativo. Nicolás del Valle, ed. Santiago: RI L eds.-U. Central. pp. 169-192.

SALINAS, C. y STANGE, H. (2009): Rutinas periodísticas. Discusión y trayectos teóricos sobre el concepto y su estudio en la prensa chilena. Cuadernos ICEI, (5). Santiago de Chile: Instituto de la Comunicación e Imagen, Universidad de Chile. (2014): La cultura profesional del periodista como mito. I Congreso de la Asociación Chilena de Investigadores en Comunicación. Pp. 320 -327. Santiago de Chile: INCOM.

SALWEN, M. (1988): Las cuatro teorías de la prensa: ¿demasiadas o insuficientes? Cuadernos. info, (4), pp. 131-141.

SAN MARTÍN, R. (2008): Contra la objetividad. El mito de la neutralidad periodística y las alternativas para repensarlo. Perspectivas de la comunicación (1), pp. 73 -80.

SCHOENBACH, K. (2001): Myths of media and audiences: Inaugural lecture as Professor of General Communication Science, University of Amsterdam. En European Journal of Communication, 16(3), pp. 361-376.

SIEBERT, F. (1984): The libertarian theory of the press. En Four theories of the press. The authoritarian, libertarian, social responsibility and Soviet communist concepts of what the press should be and do. Champaign, IL: The University of Illinois Press.

TAY LOR, R. y TEEL, L. (1985): Sala de redacción. Una introducción al periodismo. México: Gernika.

TUCHMAN, G. (1983): La producción de la noticia. Estudio sobre la construcción de la realidad. Barcelona: Gustavo Gili.

(1999): La objetividad como ritual estratégico: un análisis de las nociones de objetividad de los periodistas. Cuadernos de Información y Comunicación, $\mathrm{n}^{\circ}$ 4. pp. 199218.

VAN DIJK, T. (2000): El estudio del discurso. El discurso como estructura y proceso. Barcelona: Gedisa. 